\title{
Les conditions de vie des immigrés africains et latino-américains de
} Barcelone

Andreu Domingo I Vals

\section{Citer ce document / Cite this document :}

Domingo I Vals Andreu. Les conditions de vie des immigrés africains et latino-américains de Barcelone. In: Revue européenne des migrations internationales, vol. 12, n¹,1996. Espagne, Portugal, Grèce, pays d'immrigration. pp. 39-51;

doi : 10.3406/remi.1996.1496

http://www.persee.fr/doc/remi_0765-0752_1996_num_12_1_1496

Document généré le 07/06/2016 


\title{
Résumé
}

Les conditions de vie des immigrés d'origine africaine et latino-américaine de Barcelone

Andreu DOMINGO i VALS

L'immigration n'est pas un phénomène nouveau ni en Catalogne, ni dans la Région Métropolitaine de Barcelone. En effet l'immigration a fortement influencé le profil démographique de la région comme de la ville pendant le siècle dernier. L'immigration venue des autres régions d'Espagne, massive dans les années soixante, s'est ralentie à partir de 1975. Depuis, alors que la situation économique se détériore, on a enregistré un accroissement de la population étrangère. Les flux les plus importants viennent d'Afrique et d'Amérique Latine. Cet article propose une réflexion sur les indicateurs d'intégration à partir d'une enquête sur les conditions de vie de la population étrangère d'origine africaine dans la Région Métropolitaine de Barcelone, réalisée en 1994 par l'Institut d'Estudis Metropolitans de Barcelona.

\section{Resumen}

Las condiciones de vida de los inmigrantes de originen africana y latinoaméricana de Barcelona.

Andreu DOMINGO i VALS

La inmigración no es un fenómeno nuevo ni en Cataluña ni en la Región Metropolitana de Barcelona. De hecho, la inmigración ha conformado durante el último siglo el perfil demográfico tanto de una como de la otra. La inmigración de origen español que había llegado a ser masiva durante la década de los sesenta se ha frenado a partir de 1975. Desde entonces, y coincidiendo con la crisis económica, se ha registrado un aumento de la inmigración extranjera. Las de origen africano y latinoamericano son unas de las que más han crecido. Este articulo aborda la reflexión sobre los indicadores de integración a partir de un estudio sobre las condiciones de vida de la población extranjera de origen africano y latinoamericano en la Región Metropolitana de Barcelona, llevado a cabo durante el ano 1994 por el Instituto de Estudios Metropolitanos de Barcelona.

\begin{abstract}
The Condition of Life of Immigrants from Africa and Latin-America in Barcelona. Andreu DOMINGO i VALS

The phenomenon of immigration in Catalonia and in the Metropolitan Area of Barcelona is not new. Infact, in the last century, the immigration has been the key of the demographic system of both areas. The immigrant population from Spain, that was massive in the 60s, has diminished since 1975. Since that date, coinciding with the economical crisis, we can notice the increase of foreign immigrant population. Immigration from Africa and Latin-America is one of the immigration that has suffered a bigger increase. This paper exposes some reflections about the indicators of the integration of migrants. They stem from the analysis of the conditions of life of foreign immigrant population from Africa and Latin-Ammerica, realized in 1994 by the Metropolitan Studies Institute of Barcelona.
\end{abstract}


Revue Européenne des Migrations Internationales Volume $12-\mathrm{N}^{\circ} 1$ 1996

\title{
Les conditions de vie des immigrés africains et latino-américains de Barcelone
}

\author{
Andreu DOMINGO
}

La Région Métropolitaine de Barcelone représente 7,7\% du territoire catalan, comprend 129 communes et regroupe, selon le dernier recensement (1991), 68\% des 6.059.494 habitants de la Catalogne. Sur les 4.117.644 habitants de la Région Métropolitaine, $63,4 \%$ sont nés en Catalogne, $34,8 \%$ sont originaires du reste de l'Espagne et $1,8 \%$ seulement sont des immigrés d'origine étrangère.

Ce chiffre est sans aucun doute sous-estimé : en effet, tous les étrangers ne sont pas enregistrés, et, de plus, seules les personnes nées à l'étranger, et non les personnes de nationalité étrangère, sont comptabilisées. Mais même si on le double, ce chiffre reste de toutes façons dérisoire si on le rapproche du nombre d'immigrés venus des autres régions de l'Espagne, et très faible si on le compare à la population étrangère des autres pays européens. Alors si le nombre des immigrés étrangers est si réduit, comment s'explique l'importance qui leur est attribuée, en particulier dans la Région Métropolitaine de Barcelone?

En Catalogne, contrairement au reste de l'Espagne, l'immigration n'est pas un phénomène nouveau. Au contraire, depuis le siècle dernier, elle a été tellement importante qu'on peut la considérer comme l'élément fondamental du système démographique encore en vigueur aujourd'hui, que la démographe Ana Cabré a baptisé « système catalan de reproduction ». En effet, sur 6 millions d'habitants que compte actuellement la région, 3,6 peuvent être considérés comme le fruit, direct ou indirect de l'immigration contemporaine. La conjonction de l'accroissement naturel et de l'immigration est à l'origine d'une croissance exceptionnelle, qui a permis à la Catalogne de maintenir sa part dans la population mondiale entre $1850(1,37 \%$ ) et 1980 (1,34\%o.) (Cabré, 1991-92). Ainsi on peut considérer l'immigration comme un phénomène structurel en Catalogne. Mais, à partir des années 70 , avec la crise économique, ce phénomène se réduit et se modifie : on observe à la fois la fin des flux 
en provenance du reste de l'Espagne et l'accroissement de la population immigrée d'origine étrangère. Comment interpréter cette croissance ? L'immigration étrangère vient-elle se substituer à l'ancienne immigration espagnole ou s'agit-il de deux phénomènes complètement différents ? Se trouve-t-on devant un élargissement extrafrontalier de l'immigration ou devant une fracture du système démographique catalan, fondé sur l'immigration ?

En plus de l'importance de l'immigration dans l'ensemble catalan, il faut souligner l'inégale distribution spatiale du phénomène : la population d'origine étrangère résidant dans la Région Métropolitaine de Barcelone représente, en 1991, 73\% de la population résidant en Catalogne née à l'étranger. Etant donné le poids de l'immigration dans l'histoire récente de la Catalogne, la forte concentration de la population immigrée dans la Région Métropolitaine de Barcelone ainsi que sa tendance à s'accroître, on ne s'étonnera pas de l'intérêt qu'éveille son étude.

Depuis 1984, l'Institut d'Etudes Métropolitaines de Barcelone organise tous les cinq ans une vaste enquête sur les conditions de vie de la population de la Région Métropolitaine de Barcelone, l'Enquête Métropolitaine. Elle a pour but de mieux connaître la structure sociale et économique de la population pour agir sur la gestion urbaine et sur les conditions de vie de ses habitants (Subirats, Lozares, 1992). Dans ce contexte, l'existence de l'immigration d'origine étrangère a été perçue comme une réalité suffisamment importante pour que les renseignements sur les immigrés soient recueillis avec les mêmes objectifs. Mais, justement, l'une des principales caractéristiques de cette immigration rend la méthodologie de l'Enquête inadaptée : nous voulons parler de sa clandestinité qui fait que les immigrés échappent à tout échantillonnage et refusent tout type d'enregistrement. Ce refus explique la faible fiabilité des différents registres administratifs qui prétendent les compter, et à partir desquels on estime le stock et le flux des immigrés étrangers, quel que soit le niveau géographique de l'analyse. Pour cette raison, l'Institut d'Etudes Métropolitaines a choisi des méthodes qualitatives pour mener à bien une première approche des conditions de vie de la population immigrée. Seuls les premiers résultats de cette recherche peuvent être présentés ici, dans le cadre limité d'un article.

\section{OBJECTIFS ET MÉTHODES DE LA RECHERCHE}

Les techniques qualitatives ne permettent, ni ne prétendent offrir des résultats grâce auxquels on puisse présenter de façon représentative les conditions de vie des immigrés d'origine étrangère. Mais leur apport apparaît fondamental sur trois points:

- Elles complètent les techniques quantitatives car elles permettent de prendre en compte le caractère dynamique du processus migratoire. Ce caractère agit sur les conditions de vie ; elles sont marquées, en effet, autant par le passé que par les perspectives d'avenir, que ce soit au niveau individuel ou collectif. Les entretiens approfondis doivent permettre de recueillir des données longitudinales, complémentaires des données transversales fournies par la méthode quantitative sur laquelle se fonde l'Enquête Métropolitaine.

- Le travail qualitatif offre l'occasion de reformuler les problèmes conceptuels à propos du thème étudié, préalablement à l'approche quantitative. L'approche inten- 
sive qu'il impose donne à la recherche un aspect exploratoire qui facilite les études quantitatives ultérieures.

- Enfin, le choix d'une méthode d'entretiens approfondis permet de donner la parole aux protagonistes eux-mêmes. Les conditions de vie sont un complexe multidimensionnel, qui admet des perspectives très diverses, mais où le point de vue individuel joue un rôle très important.

Il a été décidé de limiter cette étude aux immigrés d'origine latino-américaine et africaine; ces deux groupes sont en effet ceux qui ont connu le plus fort accroissement ces dernières années, et tous les indicateurs permettent de prévoir que leur contribution à l'immigration dans la Région Métropolitaine de Barcelone gardera une place de premier plan dans les années à venir.

Parmi les multiples aspects composant le conglomérat que l'on appelle « conditions de vie », nous prendrons particulièrement en compte l'histoire migratoire, (y compris le cadre législatif), les caractéristiques familiales, les réseaux de relation et leur utilisation dans les situations difficiles, les conditions de travail et de logement, l'éducation et la langue, la perception de l'espace, enfin l'identité culturelle, y compris l'impact du racisme et de la xénophobie sur les conditions de vie des personnes interrogées.

La conception globale de la méthode part des présupposés herméneutiques exposés par Hans Georg Gadamer $(1977,82)$, c'est à dire la recherche comme un continuum ouvert à l'interrogation : interroger et être interrogé, interpréter la textualité des entretiens dans leur unité, comme produit, en analysant les réponses, mais aussi les questions des personnes chargées des entretiens. Faute de place, nous ne nous étendrons pas ici sur la justification du cadre théorique de la méthodologie, et nous limiterons à présenter de façon succinte les différentes phases de la recherche.

\section{CONFECTION DES CATÉGORIES ET GUIDE PRÉALABLE AUX ENTRETIENS}

Une typologie des personnes à interroger a été élaborée en tenant compte des variables suivantes : origine (maghrébine, subsaharienne, ou latino-américaine), sexe, situation régulière ou irrégulière au moment de l'entretien, date d'arrivée dans le pays (avant ou après 1989). Le croisement de ces variables a permis de définir 24 catégories, et pour chacune nous avons effectué trois entretiens, soit 72 au total. Afin d'étendre le spectre des personnes interrogées, nous avons ensuite pris en compte d'autres variables, comme par exemple l'âge, la distribution géographique, les contacts, le niveau d'instruction, l'activité, l'origine plus précise à l'intérieur d'un pays, la situation familiale, etc... Parallèlement à la construction de l'échantillon, nous avons rencontré divers informateurs qualifiés appartenant à des associations d'immigrés ou à des Organisations Non Gouvernementales, des responsables administratifs ainsi que des scientifiques spécialistes de la question. C'est à partir de ce premier recueil qu'a été élaboré le guide d'entretien. 


\section{LES ENTRETIENS ET LEUR CODIFICATION.}

Chaque entretien semi-directif a été mené par une seule personne, de même sexe que l'interviewé et qui lui garantissait l'anonymat absolu. Les entretiens ont été enregistrés. Une fois transcrits, ils ont été codés, en vue de leur traitement informatique. Cette codification a introduit, à son tour, une interprétation du matériel produit.

\section{EXPLOITATION.}

L'exploitation a recherché le traitement discursif, à travers la déconstruction de l'expérience autobiographique ; de ce fait, elle s'est éloignée de la méthodologie des récits de vie. Cette exploitation s'est faite à partir des critères de condition de vie énoncés plus haut.

\section{L'ÉPÉE DE DAMOCLÈS : LE CADRE LÉGISLATIF}

Les raisons invoquées par les personnes interrogées pour expliquer leur migration rejoignent celles que l'on pourrait rencontrer chez n'importe quel autre communauté de migrants; elles souhaitent améliorer leurs conditions de vie. Cette amélioration est surtout attendue d'un emploi nouveau, permettant d'améliorer leur niveau de revenus, mais l'attente porte aussi sur la formation, les conditions de travail, le suivi médical, ou l'espace de liberté pour les réfugiés politiques.

Contrairement aux immigrés provenant du reste de l'Etat espagnol, ou aux immigrés étrangers dans les autres pays européens, la plupart des immigrés extra-communautaires, africains surtout (mis à part ceux qui proviennent de l'ancienne colonie espagnole de Guinée équatoriale) n'ont pas choisi Barcelone; ils y sont arrivés car ils ne pouvaient pas accéder à d'autres pays de l'Union européenne, en particulier la France, à cause des restrictions croissantes des migrations dans ces pays. Ainsi donc, au départ, la plupart des immigrés d'origine africaine se sont installés sur le territoire de la Région Métropolitaine de Barcelone comme sur un lieu de passage, dans une méconnaissance absolue de la réalité du pays, qui est pourtant devenue leur lieu de vie. C'est à partir de ce noyau, correspondant aux immigrés arrivés dans les années 70 , que des réseaux migratoires, qui existent encore aujourd'hui, ont commencé à se constituer et à se développer. Les immigrés d'origine latino-américaine les plus récemment arrivés partagent avec les africains cette caractéristique de ne pas avoir choisi leur pays d'accueil. Ces nouveaux immigrés semblent en effet déconnectés des premiers immigrés arrivés des pays du Cône Sud pour raisons politiques, dans les années soixante-dix et quatre-vingt.

La méconnaissance préalable du pays de destination n'implique pas, pour autant, l'absence de réseaux migratoires; simplement, ils se développent en dehors de l'Espagne. En effet, le projet migratoire a été financé soit grâce à la capitalisation individuelle des revenus du travail du migrant et de sa famille dans leur pays d'origine, soit grâce à l'appui économique de membres de sa famille déjà émigrés dans d'autres pays européens. En tout cas, il semble nécessaire de replacer le processus migratoire dans la perspective des stratégies familiales.

Il faut aussi le replacer dans l'histoire des individus. Si dans le cadre espagnol l'émigration vers Barcelone peut être envisagée dans ses premiers stades, comme un 
point de départ, vue de l'extérieur de l'Espagne, elle est généralement l'aboutissement d'un processus migratoire commencé dans les pays d'origine. Cette observation est particulièrement valable pour les migrants d'origine subsaharienne (surtout du Sénégal et de Gambie), qui connaissent une grande mobilité préalable, tant dans leur pays d'origine (avec des migrations campagnes-villes), que dans les pays limitrophes, avec lesquels il existe des circuits migratoires traditionnels.

Les caractéristiques du cadre législatif conditionnent le séjour des immigrés dans le pays, ainsi que leur projet migratoire, qu'elles arrivent à bloquer. La quasi-majorité des personnes interrogées ont été à un moment en situation irrégulière, et le fait qu'elles soient arrivées à régulariser leur situation ne garantit pas qu'elles puissent conserver dans l'avenir leur statut. La lourdeur des démarches administratives le met en effet en danger. Tant que durent les démarches destinées à obtenir un permis de travail, on ne peut pas être employé régulier, ni obtenir un permis de séjour. La rigidité des critères et la durée des attentes aboutissent à des situations paradoxales comme par exemple l'arrivée du permis de travail alors que l'activité a cessé ou même la délivrance d'un permis dont la date est périmée, ce qui oblige le travailleur à recommencer l'ensemble du processus de régularisation.

Tandis que les politiques et les spécialistes débattent de la légitimité des politiques migratoires et de leurs répercussions sur l'architecture globale de la construction européenne ainsi que sur les intérêts de chacun des pays d'accueil, ou encore des relations avec les pays d'origine, le cadre législatif est un élément fondamental de la vie quotidienne des immigrés. Leur statut juridique, qui découle des politiques en matière d'immigration détermine de façon préalable leurs conditions de vie. Pour l'Etat espagnol, ce cadre législatif est constitué des lois suivantes : la Loi organique $7 / 1985$ du $1^{\text {er }}$ juillet, sur les droits et les devoirs des étrangers en Espagne, appelée généralement Ley de Extranjería (loi sur les étrangers), et plus récente, la Résolution du 7 juin 1991 du sous-secrétariat, par laquelle est publié l'accord ministériel du 7 juin 1991 sur la régularisation des travailleurs étrangers, appelée généralement la régularisation de 1991. Ces lois s'accordent avec les accords de Shengen de 1985, applicables à partir de 1991. L'une comme l'autre des deux mesures législatives espagnoles ont été critiquées, que ce soit pour leur application ou pour leur conception. De fait, on peut observer que la Loi sur les étrangers a parfois amené une dérégularisation des travailleurs immigrés dans le pays. Quant à la régularisation de 1991, elle a entraîné pour une partie de la main-d'œuvre immigrée déjà présente et indépendamment de sa durée de séjour à Barcelone un effet pervers. Lorsque ces travailleurs ont demandé à leurs employeurs du secteur informel le contrat qui leur permettait de se mettre en règle, ils ont perdu leur emploi.

Paradoxalement, le cadre législatif, au lieu d'assurer le statut de la personne immigrée et de ses activités dans le pays, introduit un élément d'incertitude, qui finit par affecter globalement toute sa vie. L'accès au marché du travail, l'accès au logement, la perception du territoire, l'espace relationnel et, en général, tout le projet migratoire, y compris dans ses effets sur la santé des personnes par la prolongation de leur précarité juridique, tout est menacé par l'épée de Damoclès de la situation juridique.

Cette menace affecte de manière contradictoire le projet de retour des personnes immigrées, qui apparaissent désorientées; elles se perdent, en effet dans un proces- 
sus d'irrésolution, dépendant de la législation même, ou vont jusqu'à inclure le durcissement des conditions légales de l'immigration dans leurs motivations pour suspendre tout projet de retour. Ainsi donc, une loi conçue pour restreindre et réguler les flux d'immigrés aboutit à les retenir, dans la clandestinité, puisque c'est sur l'immigré déjà installé et sur ses conditions de vie qu'elle a les effets les plus directs.

\section{DE LA FAMILLE À L'ETAT : FAMILLE, RÉSEAUX DE RELATIONS ET SERVICES ADMINISTRATIFS}

Comme nous lavans souligné plus haut, la dimension familiale est essentielle dans le processus migratoire. En effet, l'émigration est généralement enclenchée grâce à des chaînes migratoires, dont participent par essence les maillons des liens familiaux. De plus, l'objectif même de l'émigration est le plus souvent l'amélioration des conditions de vie de la famille, ou même la constitution d'une famille.

D'autre part, la qualité de vie, que l'on peut définir comme le degré de satisfaction des attentes de la population immigrée au sujet de ses conditions de vie, varie extraordinairement selon qu'on se réfère à une personne seule ou à toute une famille. Avec la présence d'une famille dans le pays de destination, les attentes au sujet des conditions de vie s'élargissent aux idées de chacun de ses membres; de même, la perception de l'entourage social se diversifie et se complexifie en fonction des nécessités et des perceptions individuelles de chacun. La présence de noyaux familiaux ouvre la porte à différentes voies de socialisation de la population immigrée.

Jusqu'à présent, l'immense majorité de la population immigrée d'origine africaine et latino-américaine correspondait au stéréotype du travailleur, jeune et de sexe masculin. Récemment, on a pu noter un accroissement de l'immigration féminine indépendante (surtout chez les latino-américains), mais aussi les débuts d'une immigration familiale : arrivées de femmes et d'enfants, grâce au regroupement familial ou constitution d'une famille dans le pays même. Le regroupement, parce qu'il subordonne l'arrivée des membres de la famille dans le pays d'accueil à la situation légale de la personne principale, généralement assimilée à l'homme, renforce la dépendance des conjoints, généralement les femmes, et des enfants. Cette procédure ignore le fait que souvent, le regroupement familial répond à la volonté de l'immigré de rester dans le pays et représente déjà une amélioration de ses conditions de vie, mais aussi la volonté de continuer à les améliorer grâce au travail des autres membres de la famille. Du coup, on analyse fréquemment, avec une certaine légèreté, en termes différencialistes des phénomènes parfaitement compréhensibles dans le cadre du processus migratoire, voire même provoqués par la législation. Les cas les plus courants sont la surévaluation des liens familiaux étendus, et la dépendance de la femme immigrée d'origine africaine. Dans le premier cas, l'existence de familles corésidentes de type étendue s'explique par la mobilisation maximale des liens familiaux dans les situations difficiles, ce qui réactive ou fait redécouvrir des structures familiales qui, dans d'autres circonstances, se maintiendraient de façon latente. Dans le second cas, c'est la législation même qui réduit, de façon parfois dramatique, l'ouverture sociale des femmes d'origine africaine arrivées grâce au regroupement familial, que celui ci ait été effectué de facto ou grâce à la régularisation. 
Parallèlement à l'entourage familial, les relations avec les compatriotes ainsi qu'avec la population autochtone contiennent de forts éléments de sociabilité qui pourront expliquer l'intégration des immigrés dans leur milieu social. Il faut souligner l'augmentation progressive du phénomène associatif, que l'on enregistre ces dernières années. Cette augmentation est aussi bien qualitative que quantitative, et les associations servent aussi bien de centre pour l'orientation et la défense des intérêts de la population immigrée que de lieu de dialogue, et d'espaces interculturels ouverts.

En ce qui concerne les relations avec les autochtones, il faut souligner que l'Etat assume des fonctions qui, dans le contexte socioculturel du pays d'origine sont étroitement liées au milieu familial, ce qui éclaire les relations des immigrés avec les diverses administrations. Vues sous cet angle, ces relations créent des situations ambigües, qui vont de la perception de la délimitation des fonctions comme une agression spécifique d'origine xénophobe, à la réorganisation progressive de ses propres fonctions familiales, érodées par le rôle croissant de l'Etat. Dans ce sens, ce sont les hiérarchies établies dans les sociétés d'origine qui accusent la plus grande détérioration, en particulier l'autorité masculine sur l'ensemble de la famille qui, d'un côté, est renforcée par l'administration est d'un autre côté perd de sa force.

\section{MARCHÉS ET TERRITOIRES : TRAVAIL, LOGEMENT ET PERCEPTION DU TERRITOIRE}

Sans vouloir tomber dans les explications socio-biologiques, qui proposent des métaphores fondées sur la défense territoriale de niches sociales, il apparaît clairement, à travers les réponses des personnes interrogées, qu'un lien existe entre l'accès aux marchés et la configuration territoriale. L'accès au marché du travail, l'accès au marché du logement, mais aussi l'accès au marché matrimonial, se retrouvent de façon répétée à l'origine de la concurrence entre autochtones et allochtones. Au-delà de la ségrégation territoriale, comprise comme la traduction, dans l'espace, de la discrimination sociale, cet accès aux marchés est pensé en termes d'accès et de délimitation de territoires.

L'accès aux différents marchés dont on a parlé reste, dans tous les cas, un accès segmenté, étant donné que la demande la plus forte dans le marché du travail est celle de main-d'œuvre non qualifiée. Cetțe demande n'a pas à s'ajuster au niveau d'instruction de la population immigrée, même pour les actifs autochtones cet ajustement n'est que temporaire. Sur le marché du travail, les personnes immigrées d'origine étrangère sont venues grossir la réserve de main-d'œuvre employée dans le secteur informel, qui profite du faible coût de cette main-d'œuvre, ainsi que de la tendance à le dérégularisation du marché lui-même. L'insécurité juridique pousse et maintient la population immigrée à l'intérieur de ce segment. L'activité des immigrés se caractérise par sa flexibilité, dans tous les domaines : ils passent d'une entreprise à une autre, d'un emploi régulier à un emploi irrégulier, ou changent de secteur productif, parmi ceux dont la demande est forte : construction, agriculture, textile et services. La concurrence entre immigrés, en fonction de leur origine, ne semble pas non plus exister : on remarque une forte spécialisation en fonction de l'origine, mais on peut l'attribuer, plutôt qu'au continent ou au pays, à l'origine rurale ou urbaine et au niveau 
d'instruction. Ainsi, la population d'origine subsaharienne est majoritaire dans l'agriculture, tandis que celle originaire d'Amérique Latine est prédominante dans le secteur des services.

Contrairement aux prévisions les plus optimistes, la situation actuelle ne semble pas temporaire, c'est à dire suivie d'une intégration progressive des immigrés dans d'autres segments du marché du travail. Ils sont maintenus à la limite de la précarisation, avec une forte mobilité liée à la sensibilité de leurs secteurs d'emploi à la conjoncture économique. Les contrats internes aux groupes que l'on a pu observer ces derniers temps, à l'occasion de la régularisation de 1991, correspondent, si l'on en croit les témoignages recueillis, à une dérive vers des formes d'auto exploitation, que la régularisation a pu formellement faciliter, plutôt qu'à une réelle amélioration des conditions de travail ou de revenu des personnes interrogées.

S'il existe une concurrence avec la population autochtone, on ne la trouve que dans les franges de la production marginale qui, dans l'ensemble des immigrés, occupent pour le moment une place minoritaire. Ces productions marginales peuvent aller d'activités autorisées, comme le ramassage de la ferraille, à des activités délictueuses mais semi-tolérées, comme la vente de tabac de contrebande ou la prostitution, et jusqu'à des activités prohibées, comme le trafic de drogue. Quoiqu'il en soit, ces situations, rappelons le, restent marginales, et sont favorisées par l'insécurité juridique.

Le logement, après l'emploi, doit être considéré comme la seconde grande nécessite primaire dont la satisfaction définit les conditions de vie. Il se trouve assujetti à l'emploi, à la fois par la capacité d'acquisition qu'apporte ce dernier et par les contraintes qu'entraîne sa localisation. L'accès au marché du logement est aussi restreint : d'une part il s'agit d'un sous-marché, peu attractif pour la population autochtone à cause de ses conditions matérielles, malgré le manque de logement qui caractérise la région urbaine; d'autre part la condition même d'étranger crée un marché spécifique, pour lequel on paie des prix supérieurs à ceux que paient les autochtones pour des logements comparables. A l'extrémité de la ségrégation du marché du logement on trouve la floraison nouvelle et inquiétante de bidonvilles dans certaines communes de la Région Métropolitaine de Barcelone. L'organisation territoriale de l'accès au marché du logement entraîne la ségrégation et la ghettoïsation.

Bien que le marché matrimonial en lui même ne soit pas comparable aux autres marchés, il apparaît souvent comme une forme de restriction territoriale de la population immigrée. C'est dans l'accès au marché matrimonial comme dans celui aux espaces de loisir de la jeunesse ouvrière que les attitudes discriminatoires sont le plus visibles. En témoigne le refus de certains établissements, des discothèques surtout, d'ouvrir leurs porte aux étrangers sous prétexte que les jeunes immigrés sont des foyers de tension, à cause de leur rivalité avec les autres jeunes face aux femmes présentes.

En dehors de cette discrimination clairement raciste, et heureusement isolée, l'organisation du territoire dépend de la pression policière qui s'y exerce. La référence à des quartiers, ou, en général à des espaces tranquilles ou fréquentables est une constante dans le discours des personnes interrogées et restreint l'espace dans lequel 
ils se déplacent. Ils opposent ces zones «fréquentables », où ils échappent au harcèlement policier ; aux zones trop contrôlées, qu'ils craignent de fréquenter, à cause de leur situation juridique.

La différence entre étrangers et autochtones, dans l'accès aux marchés comme dans la perception du territoire est plus accusée dans le cas des femmes, étant donné qu'elles occupent un secteur plus fragile dans le monde du travail, et que, comme nous l'avons signalé, elles sont dans une situation plus mauvaise vis-à-vis de la loi, ce qui se traduit par un accroissement des restrictions.

\section{L'IMMIGRATION ENTRE DEUX MONDES : IDENTITÉ CULTURELLE}

L'identité est une construction sociale, et en tant que telle, dynamique. La preuve en est que les personnes immigrées mettent en contact, dans leur propre expérience des cultures différentes, et qu'elles se sentent obligées, consciemment ou inconsciemment de construire leurs référents en partant de leur culture d'origine, et en gardant comme horizon socio-culturel la culture de leur milieu d'arrivée.

La perception que les personnes interrogées ont des autres communautés immigrées ou de la population autochtone, s'appuie sur des stéréotypes, dont la pauvreté conceptuelle est inversement proportionnelle à l'efficacité taxinomique. Ainsi donc, chaque personne immigrée d'origine étrangère est obligée de redéfinir sa propre identité en termes stratégiques face à des stéréotypes à caractère péjoratif, qui le rattachent à la marginalité et déprécient la réalité de sa culture d'origine. L'adoption des référents peut être complexe. Certains, souvent parmi ceux dont les situations sont les plus précaires, adoptent de façon positive la différenciation culturelle reconstruite à partir des préjugés véhiculés par la population autochtone, comme forme d'autoaffirmation. Au pôle opposé, d'autres nient leur attachement à la culture d'origine, ou en attribuent les stéréotypes négatifs à d'autres communautés, dans une lecture clairement classiste.

Enfin, les relations avec la population autochtone passent par une adaptation aux stéréotypes existants déjà sur cette population, en l'occurrence les Catalans. Ainsi, nous n'assistons pas, de la part de la population immigrée, à la création de stéréotypes nouveaux mais à la transmission de ceux qui circulent, qu'ils soient positifs ou négatifs, médiatisés par leur propre expérience personnelle. De ce point de vue, leur considération pour la langue catalane est fondamentale, cette langue étant le symbole le plus visible de l'identité et de la différence culturelle dans la société d'accueil. Le catalan est perçu et apprécié non pour sa valeur d'usage, très faible dans la population immigrée considérée, mais plutôt comme un élément de définition pour la population autochtone, et donc comme un élément stratégique face à cette population et à son identité. Les personnes interrogées ont une attitude ouverte à cet égard, une demande freinée par le manque de moyens d'apprentissage et par l'attitude linguistique de la population. De ce point de vue, leur attitude n'est pas nouvelle non plus, elle suit seulement la pente des vagues d'immigration précédentes, d'origine espagnole. L'attitude des immigrés vis-à-vis de la langue qu'ils souhaitent voir maîtriser par la deuxième génération est particulièrement significative : le choix du catalan 
peut être perçu comme une marque de volonté et d'exigence d'intégration de ses enfants, avec l'espoir que cette volonté existe aussi dans la société d'accueil.

\section{NOUVEAUX CATALANS D'ORIGINE AFRICAINE ET LATINO-AMÉRICAINE}

Les populations d'origine africaine et latino-américaine arrivées dans la Région Métropolitaine de Barcelone présentent de grandes différences sur le plan de leur insertion dans le tissu social. La majorité des immigrés d'origine africaine peut être assimilée au niveau le plus bas de la classe ouvrière, tandis que parmi les immigrés d'origine latino-américaine, on trouve des personnes qui appartiennent à la classe moyenne, et d'autres qui se répartissent dans le large éventail de la classe ouvrière. Cette différence s'explique, en termes généraux par l'époque à laquelle ils sont arrivés, mais aussi par les caractéristiques spécifiques qui ont motivé les différentes vagues migratoires. Quoiqu'il en soit, on ne doit pas oublier qu'il existe dans l'un et l'autre groupe, des exceptions significatives; comme les originaires de Guinée Equatoriale pionniers de cette immigration, ou les latino-américains des dernières décennies, certaines au niveau collectif, d'autres à caractère individuel. L'origine n'est donc pas déterminante pour les conditions de vie de la population immigrée.

Lors de la détermination des politiques visant à la régulation de l'immigration, de nombreux problèmes se posent, liés à la connaissance insuffisante du marché du travail local, de l'impact de l'immigration étrangère sur le développement des différents secteurs productifs comme du modèle de croissance économique en général, de la répercussion de la présence d'immigrés dans la société d'accueil. Le plus souvent, ces politiques n'atteignent pas l'effet recherché, étant donné que, généralement, elles sont conçues en fonction des relations bilatérales entre pays de destination et pays d'origine, ou soumises aux intérêts de grands blocs, difficilement conciliables avec les intérêts de chacun des Etats engagés. Dans la pratique, ces politiques utilisent la personne immigrée comme un élément de négociation, oscillant entre le discours policier, dans le pire des cas, et l'assistance, dans le meilleur des cas - ce qui, de fait, est complémentaire. En tout cas, les conditions de vie de la population immigrée sont compromises par de telles discussions.

Contrairement aux vagues migratoires antérieures, qui ont connu leur plus grande expansion en pleine croissance économique, les migrations actuelles se font dans une période où l'on oscille entre l'abondance et la récession économique. Le fait le plus nouveau est la coïncidence de ces mouvements (et l'offre d'emploi correspondante) avec des indices de chômage élevés parmi la population autochtone. Du même coup, la législation agit comme un frein à toute intégration possible, en faisant valoir le dangereux argument de la protection de la main-d'œuvre autochtone, ou même celui d'un supposé seuil de tolérance, dépendant du nombre d'immigrés étrangers (qui, comme nous l'avons vu est peu élevé).

$\mathrm{Si}$, comme nous l'avons montré dans cet article, l'immigration étrangère est incompréhensible sans un espace social produit antérieurement, et si le rôle de cette population ne peut, en aucun cas être considéré comme marginal, on ne doit pas non plus oublier que c'est la législation elle-même qui peut l'amener à des situations de 
marginalité, en l'empêchant son intégration. Cette intégration est indissociable de la possibilité d'un processus d'appropriation d'espaces sociaux ascendants; c'est à dire qu'il n'y a pas d'intégration possible sans mobilité sociale ascendante, sans attentes dans l'amélioration des conditions de vie, que ce soit celles de la personne immigrée, ou celles de ses enfants. La préoccupation et l'intérêt pour l'amélioration des conditions de vie des nouveaux Catalans d'origine africaine et latino-américaine affecte la globalité de la société catalane, dans un modèle de développement économique fondé sur l'intégration, tel que nous venons de le définir.

Traduit de l'espagnol par Hélène GUILLON 


\section{Références bibliographiques}

CABRE Anna «Les migracions en la reproducció de la població catalana, 1880-1980», Documents d'Analisí Geográfica, n¹9-20, pp.33-55, 1991-1992.

GADAMER Hans Georg, Verdad y metodo, Salamanca, Sígueme, 1977.

GADAMER Hans Georg, L'art de comprendre. Herméneutique et tradition philosophique, Paris, Aubier Montaigne, 1982.

SUBIRATS Marina, LOZARES Carlo (dir.), Enquesta de la Regió Metropolitana de Barcelona 1990. Condicions de vida $i$ hábits de la poblacio, Barcelone, Mancomunitat de Municipis de l'Area Metropolitana de Barcelona i Diputació de Barcelona, 1992. 
Les conditions de vie des immigrés d'origine africaine et latino-américaine de Barcelone Andreu DOMINGO i VALS

L'immigration n'est pas un phénomène nouveau ni en Catalogne, ni dans la Région Métropolitaine de Barcelone. En effet l'immigration a fortement influencé le profil démographique de la région comme de la ville pendant le siècle dernier. L'immigration venue des autres régions d'Espagne, massive dans les années soixante, s'est ralentie à partir de 1975. Depuis, alors que la situation économique se détériore, on a enregistré un accroissement de la population étrangère. Les flux les plus importants viennent d'Afrique et d'Amérique Latine. Cet article propose une réflexion sur les indicateurs d'intégration à partir d'une enquíte sur les conditions de vie de la population étrangère d'origine africaine dans la Région Métropolitaine de Barcelone, réalisée en 1994 par l'Institut d'Estudis Metropolitans de Barcelona.

\section{The Condition of Life of Immigrants from Africa and Latin-America in Barcelona.}

\section{Andreu DOMINGO i VALS}

The phenomenon of immigration in Catalonia and in the Metropolitan Area of Barcelona is not new. In fact, in the last century, the immigration has been the key of the demographic system of both areas. The immigrant population from Spain, that was massive in the 60s, has diminished since 1975. Since that date, coinciding with the economical crisis, we can notice the increase of foreign immigrant population. Immigration from Africa and Latin-America is one of the immigration that has suffered a bigger increase. This paper exposes some reflections about the indicators of the integration of migrants. They stem from the analysis of the conditions of life of freign immigrant population from Africa and Latin-Ammerica, realized in 1994 by the Metropolitan Studies Institute of Barcelona.

Las condiciones de vida de los inmigrantes de originen africana y latinoaméricana de Barcelona.

\section{Andreu DOMINGO i VALS}

La inmigración no es un fenómeno nuevo ni en Cataluña ni en la Región Metropolitana de Barcelona. De hecho, la inmigración ha conformado durante el último siglo el perfil demográfico tanto de una como de la otra. La inmigración de origen español que había llegado a ser masiva durante la década de los sesenta se ha frenado a partir de 1975. Desde entonces, y coincidiendo con la crisis economica, se ha registrado un aumento de la inmigración extranjera. Las de origen africano y latinoamericano son unas de las que más han crecido. Este articulo aborda la reflexión sobre los indicadores de integración a partir de un estudio sobre las condiciones de vida de la población extranjera de origen africano y latinoamericano en la Región Metropolitana de Barcelona, llevado a cabo durante el año 1994 por el Instituto de Estudios Metropolitanos de Barcelona. 